\title{
Cycle accurate transaction-driven simulation with multiple processor simulators
}

\author{
Dohyung Kim ${ }^{1 \mathrm{a})}$ and Rajesh Gupta ${ }^{2}$ \\ ${ }^{1}$ Engineering Center, Google Korea Ltd. \\ 737 Yeoksam-dong, Gangnam-gu, Seoul 135-984, Korea \\ 2 Dept. of Computer Science and Engineering, University of California, San Diego \\ 9500 Gilman Drive, La Jolla, CA 92093-0114, USA \\ a)dohyung@google.com
}

\begin{abstract}
Performance of multi-processor simulation is determined by how often simulators exchange events with one another and how accurately simulators model their behaviors. Previous techniques have limited their applicability or sacrificed accuracy for performance. In this paper, we notice that inaccuracy comes from events which arrive between event exchange boundaries. We propose cycle accurate transaction-driven simulation which maintains event exchange boundaries at bus transactions but compensates for accuracy. The proposed technique is implemented in CATS framework and our experiment with 64 processors achieves $1.2 \mathrm{M}$ processor cycles/s.
\end{abstract}

Keywords: multi-processor, simulation, transaction-level model Classification: Integrated circuits

\section{References}

[1] J. Jung, S. Yoo, and K. Choi, "Performance improvement of multiprocessor systems cosimulation based on SW analysis," DATE 2001, pp. 749-753, March 2001.

[2] D. Kim, Y. Yi, and S. Ha, "Trace-Driven HW/SW Cosimulation Using Virtual Synchronization Technique," DAC 2005, Anaheim, June 13-17 2005.

[3] C. Lennard and D. Mista, "Taking Design to the System Level," the white paper for SoC Designer, a ARM Inc.

[4] CATS Framework, [online] http://mesl.ucsd.edu/dhkim/CATS

[5] D. Burger and T. M. Austin, "The SimpleScalar Toolset, Version 2.0," Univ. of Wisconsin-Madison CS Dept., Technical Report \#1342, June 1997. 


\section{Introduction}

We identify two main factors which affect multiprocessor simulation performance. First, a multiprocessor simulator usually has a single code to model processor behavior and switches different processor instances. Thus, when we invoke a processor, the instance switch generates additional overhead. Second, we have to advance clocks of processor simulators and an inter-connection network simulator while exchanging events at every clock.

When optimizing those two factors, granularity and abstraction of simulators have major effects to simulation performance. Granularity indicates how frequently a simulator exchanges events with other simulators. Abstraction determines how accurately a simulator models behavior of a processor or an inter-connection. If we increase granularity, the number of instance switches is reduced. If we have higher abstraction, it reduces time to advance clocks of simulators.

\section{Background}

Previous approaches have utilized granularity and abstraction to overcome performance problems of multiprocessor simulation. Software analysis predicts [1] arrival times of events by statically analyzing instructions of other simulators. If we know when the next event arrives at a simulator, we can safely advance the clock of the simulator until that time. Virtual synchronization [2] suspends to process events to a simulator by analyzing behavior of the simulator as long as events do not affect behavior of the simulator. Transaction level model (TLM) [3] abstracts behavior of a simulator by simplifying transitions through multiple states between abstraction boundaries.

All three approaches try to enlarge granularity between boundaries. However, the first and the second approaches have limited their applicability because a static analysis does not predict dynamic behaviors of caches or operating systems. The third approach may sacrifice accuracy for performance.

We notice that three approaches are complementary and propose a novel cycle accurate transaction-driven simulation (CATS) technique. The CATS technique improves simulation performance while maintaining cycle accuracy.

\section{Cycle accurate transaction-driven simulation}

CATS enlarges granularity of simulators to bus transaction and abstract behavior of a shared bus at bus transaction level similar to TLM. However, events which arrive between granularity boundaries may cause inaccuracy.

Fig. 1 shows an example of such a bus transaction. In the transaction, the processor 1 reads data in the modified cache line of the processor 2 and the processor 3 requests a shared bus. When the processor 1 sends the first address of the cache line, the processor 2 interrupts behavior of the shared bus and provides data to the processor 1 instead of a memory. Moreover, the shared bus has to handle the bus request event from the processor 3 .

Fig. 2 shows an execution sequence of simulators applying CATS, which processes the bus transaction in Fig. 1. Upper figures show what events each 


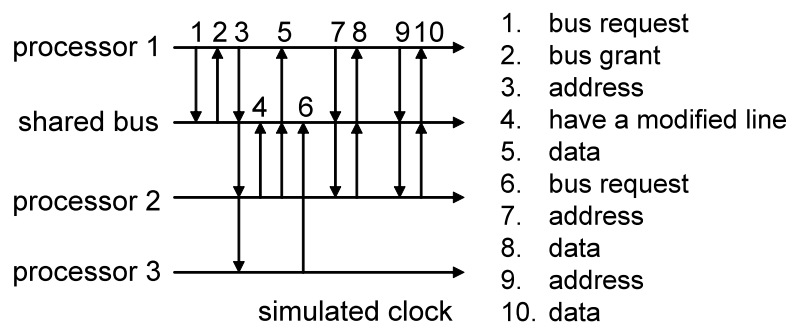

Fig. 1. An example of a bus transaction

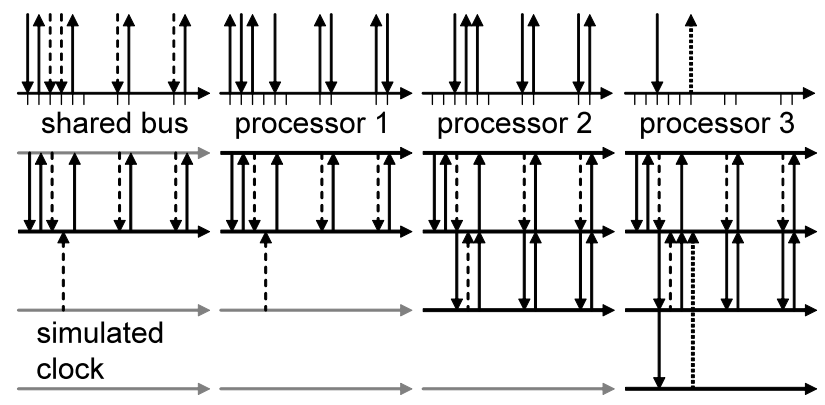

$\rightarrow$ predicted event $\rightarrow . . . . \rightarrow$ suspended event

Fig. 2. An execution sequence of simulators applying CATS

simulator has during the bus transaction. Bottom figures illustrate how each execution of a simulator incrementally appends events to the bus transaction.

Before CATS advances clocks of simulators, it examines behavior of processors to predict events to the shared bus during the bus transaction. Unlike previous approaches, CATS not only predict arrival times of events but also values of them. Thus the shared bus simulator can advance its clock without interactions with other processors. In addition, behavior of the shared bus enables CATS to suspend a certain type of events to the shared bus. In the example, CATS can predict event 3, 4, 7 and 9, and suspend the event 6 .

With predicted events, first, CATS advances the clock of the shared bus until the shared bus finishes processing the bus transaction. During the execution, CATS captures timings of events. Then when CATS advances the clocks of processors, the timings enable processors to emulate incoming events from the shared bus.

Compared to Fig. 1, Fig. 2 shows an identical simulated result except that CATS does not notify the arrival of the event 6 to the shared bus. However, because we know that the shared bus can suspend the event 6 , the difference does not hurt cycle accuracy.

In following sections, we explain the proposed technique in detail. Section 3.1 shows how CATS predicts and suspends events. Section 3.2 introduces how to capture timings of events and handle those timings at processors. 


\subsection{Handling events to a shared bus}

A shared bus receives bus request events, address events (or address events with data) and cache coherence events from processors.

First, if a bus transaction locks the bus, the bus arbiter performs arbitration at the end of the bus transaction. Otherwise, the bus arbiter performs arbitration at the end of every memory transaction of the bus transaction.

CATS utilizes such behavior of the bus arbiter. If the locked flag is set, CATS suspends bus request events until the shared bus finishes processing the bus transaction. Otherwise, it suspends requests until one memory transaction ends.

Second, when a processor receives a bus grant, it sends the first address of a bus transaction at the next bus cycle. If the processor receives a data and burst accesses of the bus transaction are not finished, it sends the next address at the next bus cycle. With this property of processors, CATS predicts arrival times and values of address events.

Third, when a processor watches an address which the processor has in its cache line, it sends a cache coherence event to a shared bus and then the shared bus changes its behavior to handle the event. For example, when the shared bus meets a read access to a modified cache line (or a shared cache line), the shared bus makes a requesting processor read data from a processor with that cache line instead of a memory. For a write access to a modified cache line, the shared bus makes a requesting processor first read data from the processor with the cache line and then send a write invalidate event (or update data) to all processors with the cache line.

CATS examines all caches of processors for the cache line in request before a shared bus processes a bus transaction. If CATS finds that a processor has that cache line, it predicts that the processor with the cache line will send a cache coherence event after it watches the first address to access. Therefore, CATS transforms the bus transaction to notify the arrival of the cache coherence event.

\subsection{Handling events to processors}

We can model behavior of the shared bus cycle accurately due to techniques in section 3.1, which also means that we can have accurate timings of outgoing events. Based on this property, we revise a behavior model of the shared bus to store timings of occurred events and pack them into a reply to a bus transaction. In other word, the model only abstracts behavior, not timings of events. Because the simulation kernel advances the clock of the shared bus prior than those of processors, processors acquire exact arrival times of incoming events with the captured timings.

When a processor receives the reply from the shared bus, the processor utilizes the timings in different ways based on event types. The events can be interrupt events from other processors, events related to cache coherence and data events from the shared bus.

First, interrupt events from other processors are delivered through a shared bus. The bus can be a dedicated bus or shared by instruction and 
data accesses. For simplicity, in this paper, we assume that interrupt events share a bus with other accesses. Thus, when a processor sends an interrupt event to other processor, the event is delivered through the shared bus to an interrupt handler. Then the interrupt handler sends an interrupt signal to a destination processor.

Therefore, if a processor watches transactions to interrupt handlers on the shared bus, the processor knows when an interrupt event arrives or it does not. If the processor does not receive an interrupt signal, the processor advances its clock until the current transaction ends. Otherwise, the processor receives the interrupt signal when a static delay is passed after a data is written to the handler.

Second, when a processor writes data to a shared cache line, processors with the cache line invalidate or update the cache line as soon as the processors watch the first address at the shared bus.

Finally, if a processor supports an early resuming of pipeline stages, the processor does not need to suspend its pipeline stages at the end of the bus transaction. Instead, the processor resumes its pipeline stages with the first data of the cache line.

\section{Experiments}

We have implemented the proposed technique in a CATS framework [4] based on SimpleScalar [5]. The CATS framework configures different architecture platforms by using an architecture description script and provides programming interfaces for a multithreaded program model. Currently the CATS framework supports ARM processors connected with AMBA AHB buses. We have also ported four programs from SPLASH-2 benchmark on the multithreaded program model in CATS: fast Fourier transform (FFT), Cholesky factorization (CHOLESKY), radix sorting (RADIX) and volume rendering (VOLREND).

In experiments, we use Fedora Linux core 3 with Pentium-M $1.7 \mathrm{GHz}$ CPU. For simulations, we assume that processors and a memory are connected to a shared bus. Each processor has separated L1 caches with $8 \mathrm{~KB}$ each and a unified L2 cache with $64 \mathrm{~KB}$.

First, we evaluate efficiency and accuracy of the CATS framework compared to an original SimpleScalar ARM with the programs from SPLASH-2 benchmark. In the experiments, memory delays are set to 18 processor cycles in non-sequential accesses and 2 processor cycles in sequential accesses.

Simulation performances of CATS are close to $80 \%$ of the SimpleScalar ARM, which means that CATS effectively reduces all components of simulation time except behavior model of processors. Errors in simulated cycles are under $0.003 \%$ except $0.13 \%$ of FFT when we extend a static delay model of the SimpleScalar ARM to an inter-connection network simulator using CATS.

Second, we measure simulation performances by changing the number of processors from one processor to sixty-four processors. The benchmark 


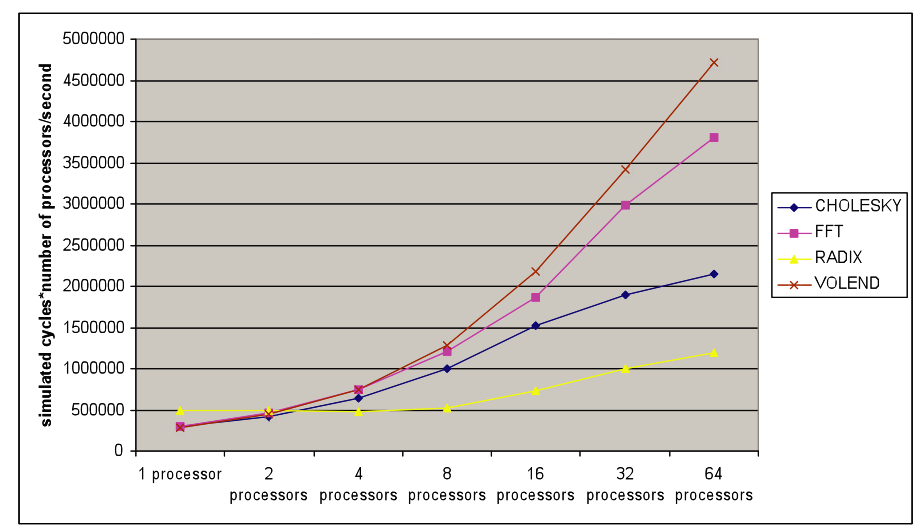

Fig. 3. Simulation performances with simulated cycles * number of processors / second

programs are automatically parallelized on the processors when we specify the number of processors at the start of the programs. However, a single bus cannot handle memory accesses from sixty-four processors. Thus we assume an ideal bus where a shared bus and processors have the same clock period, and all memory delays are one cycle.

In Fig. 3, we calculate simulation performances by multiplying the number of processors to simulated cycles per second, i.e., processor cycles/s. We notice that changing number of processors even increases simulation performances in CATS depending on program characteristics. When a program has more memory transactions, we can see more performance gain.

In CATS, more memory transactions enlarge granularities of event exchanges in both the inter-connection and processor simulators because we exchange events between bus transaction boundaries. In addition, higher abstraction of the inter-connection simulation does not suffer from increased memory transactions. Longer memory latencies due to memory conflicts require fewer computations by decreasing IPC (instructions per cycle) in processor simulators. As a result, CATS can perform better with more processors.

\section{Conclusion}

We have shown that event predictions to a shared bus can enable us to advance the clock of the shared bus without interactions with processors. This is possible based on analysis of the processor simulators. The behavior model of the shared bus stores timings of events and provides cycle accuracy to processor simulators. The proposed technique is implemented in the CATS framework. The framework provides simulation performances comparable to TLM with cycle accuracy. As future work, we are working to provide additional processor and inter-connection network models. We will examine accuracy with multiple processors by comparisons with a real multi-processor system. 


\section{Acknowledgments}

Our work was supported by a gift from Intel Corporation, a grant from UC Discovery program and IT scholarship program which is supervised by Institute for Information Technology Advancement \& Ministry of Information and Communication, Republic of Korea. 